\title{
Critical Loads and Dynamic Modelling to Assess European Areas at Risk of Acidification and Eutrophication
}

\author{
J.-P. Hettelingh • M. Posch • J. Slootweg • \\ G. J. Reinds $\cdot$ T. Spranger $\cdot$ L. Tarrason
}

Received: 17 June 2005 /Revised: 2 December 2005 / Accepted: 17 December 2005 / Published online: 30 January 2007

(C) Springer Science + Business Media B.V. 2007

\begin{abstract}
European critical loads and novel dynamic modelling data have been compiled under the LRTAP Convention by the Coordination Centre for Effects. In $20009.8 \%$ of the pan-European and $20.8 \%$ of the EU25 ecosystem area were at risk of acidification. For eutrophication (nutrient $N$ ) the areas at risk were 30.1 and $71.2 \%$, respectively. Dynamic modelling results reveal that $95 \%$ of the area at risk of acidification could recover by 2030 provided acid deposition is reduced according to present legislation. Insight into the timing of effects of exceedances of critical loads for nutrient $N$ necessitates the further development of dynamic models.
\end{abstract}

J.-P. Hettelingh $(\bowtie) \cdot$ M. Posch $\cdot$ J. Slootweg Coordination Centre for Effects, Netherlands Environmental Assessment Agency (MNP), P.O. Box 303, NL-3720 AH Bilthoven, The Netherlands e-mail: j.p.hettelingh@mnp.nl

G. J. Reinds

Alterra, Wageningen University and Research Centre (WUR),

P.O. Box 47, NL-6700 AA Wageningen, The Netherlands

T. Spranger

Umweltbundesamt (UBA),

P.O. Box 1406, 6813 Dessau, Germany

L. Tarrason

EMEP/MSC-W, Norwegian Meteorological Institute,

P.O. Box 43, Blindern,

0313 Oslo, Norway
Keywords acid deposition · critical loads · dynamic modelling $\cdot$ exceedances $\cdot$ LRTAP Convention

\section{Introduction}

The critical load concept has been developed in Europe since the mid-1980s, mostly under the auspices of the 1979 Convention on Long-range Transboundary Air Pollution (LRTAP). European data bases and maps of critical loads have been instrumental in formulating effects-based Protocols to the LRTAP Convention, such as the 1994 Protocol on Further Reduction of Sulphur Emissions (the 'Oslo Protocol') and the 1999 Protocol to Abate Acidification, Eutrophication and Ground-level Ozone (the 'Gothenburg Protocol'). The methods and maps used to support these agreements are described in Hettelingh, Posch, De Smet, Downing (1995) and Hettelingh, Posch, De Smet (2001).

In 2005, European data on critical loads for acidification and eutrophication was updated, including a novel database on dynamic modelling outputs. By definition, critical loads do not provide any information on time scales of recovery or damage. Therefore, the use of dynamic models has been recognized as an important part of the work programme under the LRTAP Convention. Of particular interest are the ecosystems where depositions exceeded or continue to exceed critical loads. 
This paper focuses on the dynamic modelling results. It also summarizes the European critical load database and provides information on the extent of their exceedances.

\section{Materials and Methods}

\subsection{Critical Loads}

Most of the European critical loads are computed with the Simple Mass Balance (SMB) (or related) models (Sverdrup \& De Vries, 1994), applied by the Coordination Centre for Effects (CCE) and a network of 25 National Focal Centres associated with the Modelling and Mapping Programme under the LRTAP Convention. The methods are described in a Mapping Manual (UBA, 2004); they have not significantly changed since they were summarized in Hettelingh et al. (2001).

Critical loads are compared to depositions of sulphur and nitrogen, computed with the EMEP 'unified' longrange atmospheric transport and dispersion model (Tarrasón et al., 2005), to compute the percentage of ecosystem area exceeded or the exceedance amounts in the $50 \times 50 \mathrm{~km}^{2}$ EMEP grid cells covering Europe. Exceedances are expressed as so-called average accumulated exceedance, the area-weighted mean of individual critical load exceedances within a grid cell (see Posch, Hettelingh, \& De Smet, 2001).

\subsection{Dynamic Modelling}

Models to determine critical loads consider only the steady-state condition for a prescribed chemical and biological response. Dynamic models, on the other hand, attempt to estimate the time required for a new steady state to be achieved. Over the past 20 years dynamic soil-chemical models have been applied to a large number of individual sites, and there are also several examples of earlier applications on a regional scale. In view of the broad published knowledge on dynamic modelling, a methodological summary will not be attempted here; a description of the most important processes captured in dynamic models can be found in UBA (2004).

In critical load assessments, two cases can be distinguished when comparing critical loads to deposition: (1) the deposition is below critical loads, and
(2) the deposition is greater than critical loads. In the first case there is no apparent problem; in the second case there is, by definition, an increased risk of damage to the ecosystem, and deposition should be reduced. However, it is often assumed that reducing deposition to critical loads immediately removes the risk of 'harmful effects,' i.e. the chemical criterion that links the critical load to the biological effects, immediately attains a non-critical value. But the reaction of soils, especially their solid phase, to changes in deposition is delayed by finite buffers, such as the cation exchange capacity. It might take decades or even centuries, before steady state is reached. Therefore, dynamic models are needed (a) to estimate the time involved in attaining a certain chemical state in response to emission scenarios, or conversely (b) to estimate so-called target loads deposition levels to be achieved through sufficient emission reductions - at which recovery will occur in a specified year (the target year). In this paper results for the target years 2030 and 2100 are reported.

Figure 1 summarises the possible development of a (soil) chemical and biological variable in response to a 'typical' temporal deposition pattern. Five stages can be distinguished (see Posch, Hettelingh, \& Slootweg, 2003):

- Stage 1: Deposition was and is below the critical load (CL), e.g. in the pre-industrial era, and the chemical and biological variables do not violate their respective criteria.

- Stage 2: Deposition is above the CL, but (chemical and) biological criteria are not violated because there is a time delay before this happens. Therefore, no damage is likely to occur at this stage. The time between the first exceedance of the CL and the first violation of the biological criterion (the first occurrence of actual damage) is termed the 'Damage Delay Time' $\left(\mathrm{DDT}=t_{3}-t_{1}\right)$.

- Stage 3: The deposition is above the CL and both the chemical and biological criteria are violated. Measures are required to avoid a (further) deterioration of the ecosystem status.

- Stage 4: Deposition is below the CL, but the criteria are still violated and thus recovery has not yet occurred. The time between the first non-exceedance of the CL and the subsequent non-violation of both criteria is termed the 'Recovery Delay Time' (RDT= $\left.t_{6}-t_{4}\right)$. 


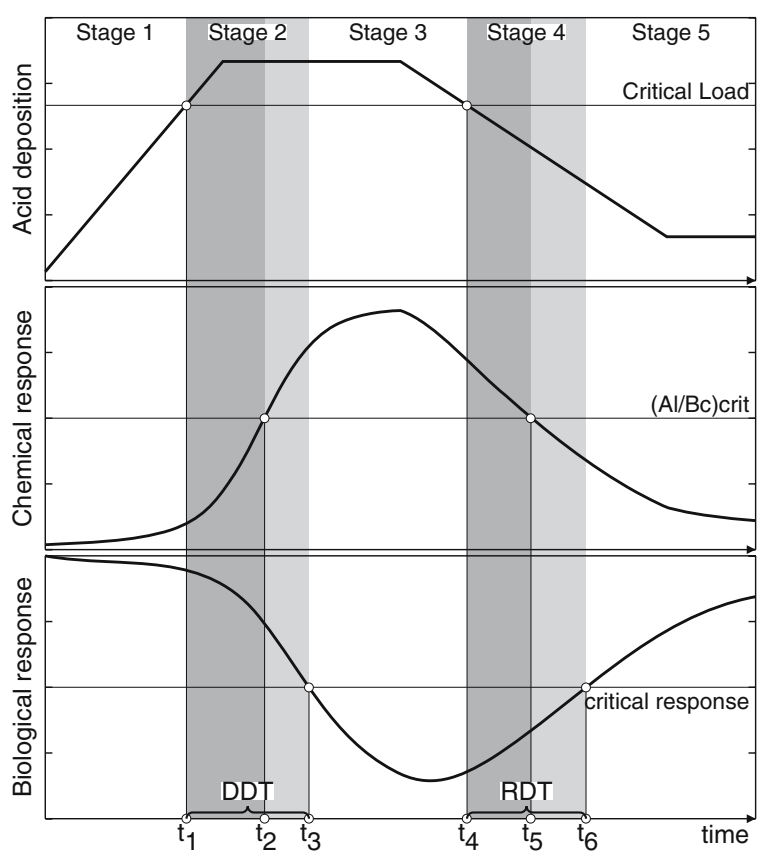

Fig. 1 'Typical' past and future development of the acid deposition (top) and its effects on a soil chemical variable (middle) and the corresponding biological response (bottom) in comparison to the critical values of those variables. The delay between the (non-)exceedance of the critical load, the (non-) violation of the critical values is indicated in grey shadings, highlighting the Damage Delay Time (DDT) and the Recovery Delay Time (RDT) of the system (from Posch et al., 2003)

- Stage 5: Deposition is below the CL and both criteria are no longer violated; and only at this stage can the ecosystem be considered to have recovered. Stages 2 and 4 can each be subdivided into two sub-stages: Chemical delay times $\left(\mathrm{DDT}_{\mathrm{c}}=t_{2}-t_{1}\right.$ and $\mathrm{RDT}_{\mathrm{c}}=t_{5}-t_{4}$; dark grey in Fig. 1) and (additional) biological delay times $\left(\mathrm{DDT}_{\mathrm{b}}=t_{3}-t_{2}\right.$ and $\mathrm{RDT}_{\mathrm{b}}=t_{6}-t_{5}$; light grey).

Dynamic modelling was carried out with a deposition path resulting from the Current Legislation (CLe) emissions scenario, which implements the Gothenburg Protocol and the National Emission Ceilings Directive of the European Union.

\section{Results}

\subsection{European Critical Loads and Exceedances}

Figure 2 shows maps of critical loads for different ecosystem classes, based on national contributions from 25 countries. For countries that never submitted critical loads, data from the European background database have been used (Posch, Slootweg, \& Hettelingh, 2005).

The maps show for every EMEP grid cell the 5th percentile of the critical load for acidification for all ecosystems combined (top-left), forest ecosystems (top-right), semi-natural vegetation (bottom-left) and aquatic ecosystems. Low critical loads below $200 \mathrm{eq}$ $\mathrm{ha}^{-1} \mathrm{yr}^{-1}$ (red shaded) occur mostly in the northern parts of Europe. While in Norway only surface waters are sensitive, in Sweden both forest and aquatic ecosystems have low critical loads. In the United Kingdom most of the sensitive ecosystems are classified as semi-natural vegetation. Similar maps for the critical loads for eutrophication (not shown here; but see Posch et al., 2005) show values for the 5 th percentile below 400 eq $\mathrm{ha}^{-1} \mathrm{yr}^{-1}$ ) in large parts of Europe.

Using EMEP depositions for the year 2000, maps of exceedances of critical loads for all ecosystems are shown in Fig. 3. In Europe $8.5 \%$ of the ecosystem area is at risk of acidification (and $17.5 \%$ for the EU25), whereas for eutrophication the area with exceedances covers $28.5 \%$ of the ecosystem area (65.2\% for the EU25). The areas where exceedances occur are of most interest for the application of dynamic models.

\subsection{Dynamic Modelling Results}

Table 1 summarizes the dynamic modelling results. Most relevant is the ecosystem area where critical loads are exceeded. Using the data from Fig. 3, this area turns out to be $579,975 \mathrm{~km}^{2}$. The area for which dynamic modelling has been carried out is $683,237 \mathrm{~km}^{2}$ (see column 2 in Table 1), and this area includes most of that exceeded area. Of the area in column 2, 168,661 km² turned out not to be safe (column 3), meaning that the critical loads are exceeded or that the critical limit is violated (or both). All following columns are expressed as percentages of this 'non-safe' area.

Column 4 shows the percentage of the area for which a Recovery Delay Time (RDT) can be computed under the CLe scenario. This is the case for ecosystems for which the critical load is at present no longer exceeded, but the critical limit is still violated (see Fig. 1). It shows that in Europe 29.2\% of the area, which is not safe at present, would recover 

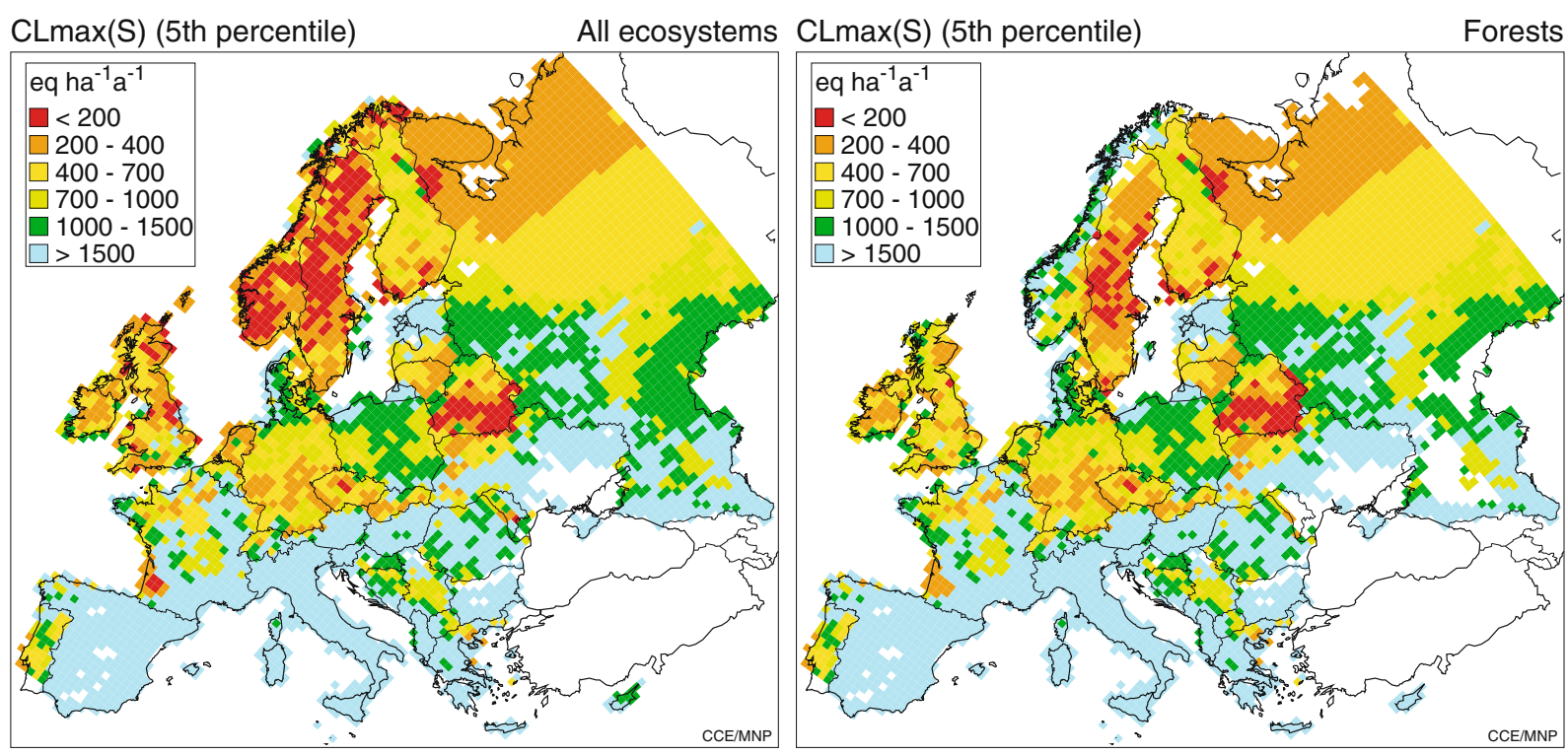

CLmax(S) (5th percentile)
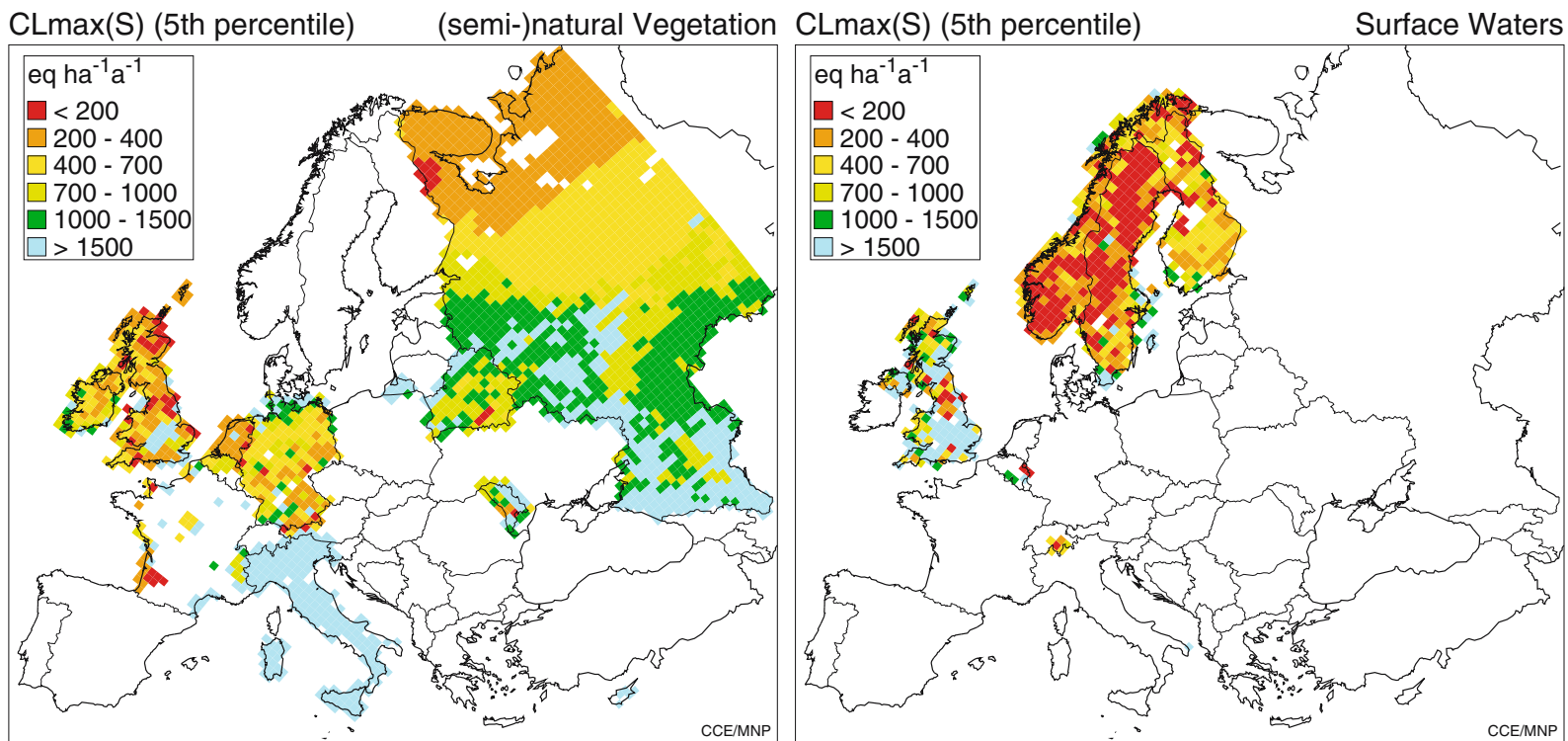

Fig. 2 The 5th percentile of the critical loads for acidification for all ecosystems (top left), forests (top right), semi-natural vegetation (bottom left) and surface waters

sometime in the future without further emission reductions. In fact, CLe depositions cause $20.2 \%$ to recover already before 2030 (see column 6), while by 2100 they will lead to a recovery of $22.3 \%$ of the ecosystem area (column 10). Comparing column 10 to 4 , we conclude that $29.2-22.3=6.9 \%$ of the area which is not safe at present would recover only after 2100. Deposition levels would need to be reduced to enlarge the area that recovers before 2100 or to bring closer the year of recovery. By how much deposition has to be reduced to obtain the target load, depends on the year (the target year) in which recovery should occur. The percentage of the European area for which target loads can be computed in 2030 and 2100 are provided in columns 8 , and 12 , respectively.

Column 5 gives the percentage of the area for which a Damage Delay Time (DDT) can be computed (see Fig. 1). This is the case in areas where the critical load is already exceeded, but the critical limit is not yet violated. In Europe $23.4 \%$ of the non-safe ecosystem area (column 3) will be damaged in the future under the CLe scenario. 

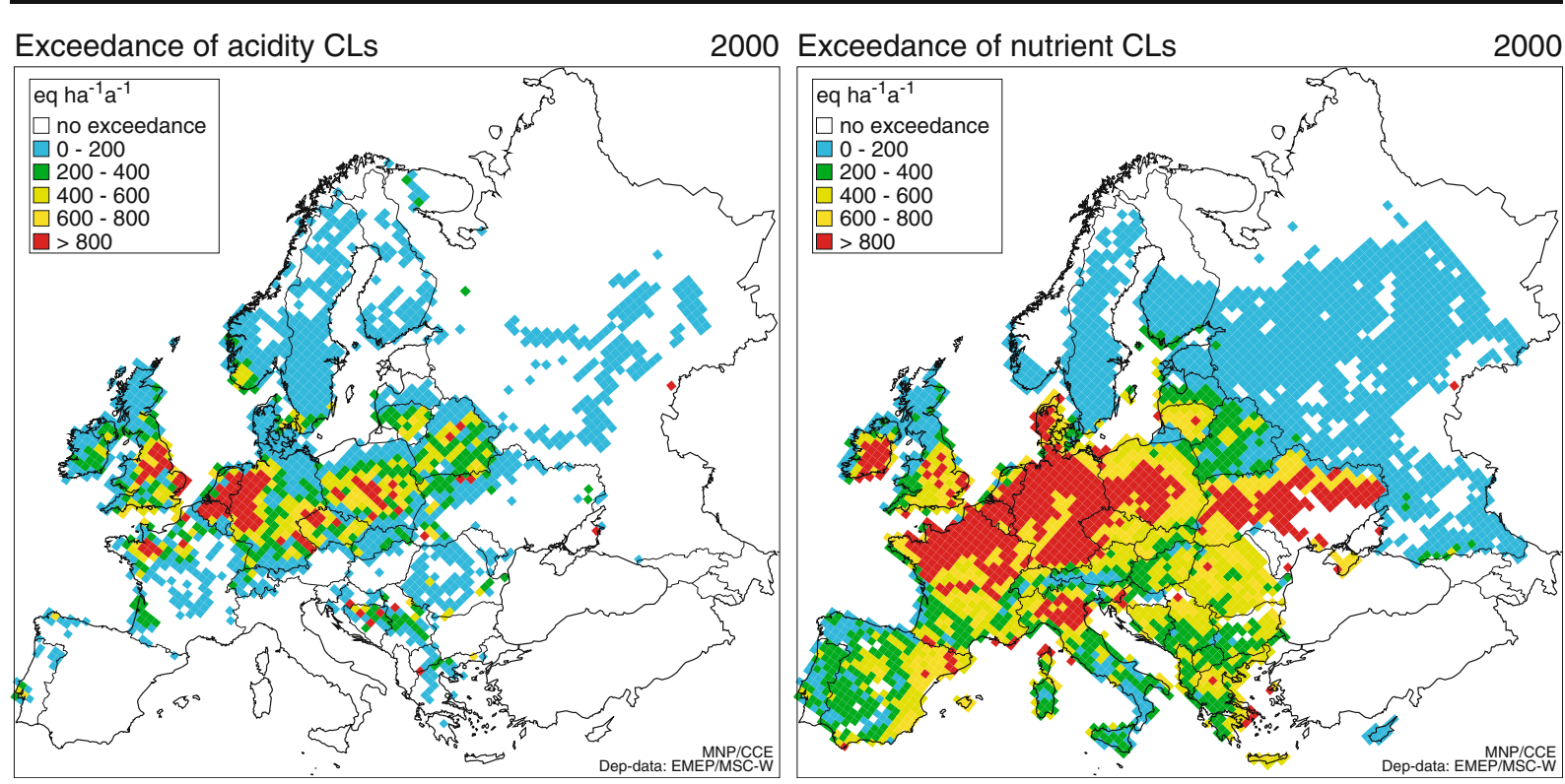

Fig. 3 Average accumulated exceedance for all ecosystems in the year 2000 of acidity (left) and nutrient $N$ critical loads. White areas indicate non-exceedance or no data

Column 6 gives the percentage of the area that will be safe (critical limit not violated and deposition not exceeding critical loads) in 2030 under the CLe scenario, i.e. $20.2 \%$. Column 7 lists the percentages of areas at risk (not safe) where target loads for recovery in 2030 equal critical loads, i.e. $24.2 \%$ in Europe. Target loads lower than critical loads (column
8) are found for $50.7 \%$ of the ecosystem area. The area for which no target loads can be found, i.e. for which even zero deposition would not lead to recovery in 2030, covers 5\% in Europe (column 9). We conclude that the area which is - and would become - safe in 2030 (columns $6+7+8$ ) is about $95 \%$ of the areas which are not safe now (column 3 ).

Table 1 Summary of dynamic modelling results (see text for explanations)

\begin{tabular}{|c|c|c|c|c|c|c|c|c|c|c|c|c|}
\hline \multirow{3}{*}{$\begin{array}{l}1 \\
\text { Country }\end{array}$} & \multirow{3}{*}{$\begin{array}{l}2 \\
\text { DynMod } \\
\mathrm{km}^{2}\end{array}$} & \multirow{3}{*}{$\begin{array}{l}3 \\
\text { Non-safe } \\
\mathrm{km}^{2}\end{array}$} & \multirow{3}{*}{$\begin{array}{l}4 \\
\text { RDT } \\
\%\end{array}$} & \multirow{3}{*}{$\begin{array}{l}5 \\
\text { DDT } \\
\%\end{array}$} & 6 & 7 & 8 & 9 & 10 & 11 & 12 & 13 \\
\hline & & & & & \multicolumn{4}{|c|}{ Target year 2030} & \multicolumn{4}{|c|}{ Target year 2100} \\
\hline & & & & & Safe & $\mathrm{TL}=\mathrm{CL}$ & TLs & n.f. & Safe & $\mathrm{TL}=\mathrm{CL}$ & TLs & n.f. \\
\hline AT - Austria & 35,745 & 334 & 31.0 & 53.4 & 31 & 36.4 & 32.6 & 0 & 31 & 36.4 & 32.6 & 0 \\
\hline $\mathrm{BG}$ - Bulgaria & 47,887 & 0 & 0 & 0 & 0 & 0 & 0 & 0 & 0 & 0 & 0 & 0 \\
\hline $\mathrm{CH}$ - Switzerland & 11,612 & 2,650 & 14.8 & 0 & 9 & 24.5 & 63.8 & 2.7 & 13.7 & 26.3 & 59.9 & 0 \\
\hline CZ - Czech Republic & 11,178 & 8,004 & 27.1 & 14.9 & 22.2 & 13.6 & 59.2 & 4.9 & 25 & 13.6 & 60.7 & 0.7 \\
\hline DE - Germany & 104,195 & 57,639 & 23.7 & 16.7 & 21.6 & 17.2 & 58.7 & 2.5 & 22.7 & 16.5 & 59.4 & 1.5 \\
\hline FR - France & 180,074 & 21,510 & 38.9 & 15.3 & 35.8 & 16 & 43.5 & 4.7 & 38.6 & 16 & 44.1 & 1.3 \\
\hline GB - United Kingdom & 1,190 & 401 & 83.8 & 0 & 16.2 & 7 & 59.2 & 17.6 & 16.2 & 16.4 & 53.9 & 13.6 \\
\hline HU - Hungary & 10,448 & 0 & 0 & 0 & 0 & 0 & 0 & 0 & 0 & 0 & 0 & 0 \\
\hline IE - Ireland & 8,936 & 1,542 & 42.3 & 41.7 & 42.3 & 44.3 & 13.4 & 0 & 42.3 & 44.5 & 13.1 & 0 \\
\hline IT - Italy & 125,878 & 0 & 0 & 0 & 0 & 0 & 0 & 0 & 0 & 0 & 0 & 0 \\
\hline NL - Netherlands & 6,052 & 3,984 & 1.3 & 4.3 & 0 & 14.2 & 71.7 & 14.2 & 1.3 & 14.3 & 84.4 & 0 \\
\hline NO - Norway & 20,535 & 12,183 & 76.5 & 0 & 0 & 7.5 & 87.6 & 4.9 & 0 & 13 & 86.4 & 0.7 \\
\hline PL - Poland & 88,383 & 48,739 & 19.8 & 47.8 & 19.2 & 47.5 & 32 & 1.2 & 19.8 & 46.7 & 33.5 & 0 \\
\hline SE - Sweden & 31,124 & 11,676 & 38.5 & 8.8 & 13.9 & 2.4 & 52.3 & 31.4 & 29.1 & 2.2 & 41 & 27.8 \\
\hline Europe & 683,237 & 168,661 & 29.2 & 23.4 & 20.2 & 24.2 & 50.7 & 5 & 22.3 & 24.2 & 50.8 & 2.7 \\
\hline
\end{tabular}


Finally, 10-13 provide the analogous information for 2100. Note, that the areas defined as 'safe' (columns 6 and 10) increase from 2030 to 2100 , whereas the areas for which target loads are not feasible (columns 9 and 13) diminish in the same period. This implies that part of the area that could recover in 2030 by identifying a target load would recover by 2100 under the CLe scenario, i.e. without additional reduction measures.

\section{Conclusions and Recommendations}

Using depositions for the year 2000 shows that critical loads in $8.5 \%$ of the ecosystem area in Europe are exceeded; and for eutrophication this area is even $28.5 \%$. These exceedances imply that those ecosystems are sooner or later at risk of being damaged. To be able to estimate the timing of this damage, dynamic modelling is required. The use of dynamic models of acidification enabled to identify that about $95 \%$ of the ecosystems still at the risk of damage in 2010 could recover by 2030 if acid deposition is sufficiently reduced. This includes about $50 \%$ of the ecosystems for which reductions even below critical load are required. The high exceedances of critical loads for eutrophication make it desirable to use dynamic models to improve also our knowledge on time delays of damage and recovery caused by excessive inputs of nitrogen.

\section{References}

Hettelingh, J.-P., Posch, M., \& De Smet, P. A. M. (2001). Multi-effect critical loads used in multi-pollutant reduction agreements in Europe. Water, Air and Soil Pollution, 130, 1133-1138.

Hettelingh, J.-P., Posch, M., De Smet, P. A. M., \& Downing, R. J. (1995). The use of critical loads in emission reduction agreements in Europe. Water, Air and Soil Pollution, 85, 2381-2389.

Posch, M., Hettelingh, J.-P., \& De Smet, P. A. M. (2001). Characterization of critical load exceedances in Europe. Water, Air and Soil Pollution, 130, 1139-1144.

Posch, M., Hettelingh, J.-P., \& Slootweg, J. (eds.) (2003). Manual for dynamic modelling of soil response to atmospheric deposition. Coordination Center for Effects, RIVM Report 259101012, Bilthoven, Netherlands, 71 pp (http://www.mnp.nl/cce).

Posch, M., Slootweg, J., \& Hettelingh, J.-P. (Eds.) (2005). European critical loads and dynamic modelling results, Status Report 2005. Coordination Center for Effects, MNP-Report 259101016, Bilthoven, Netherlands, 167 pp (http://www.mnp.nl/cce).

Sverdrup, H., \& De Vries, W. (1994). Calculating critical loads for acidity with the simple mass balance methods. Water, Air and Soil Pollution, 72, 143-162.

Tarrasón, L., Benedictow, A., Fagerli, H., Jonson, J. E., Klein, H., Van Loon, M., et al. (2005). Transboundary acidification, eutrophication and ground level ozone in Europe in 2003, EMEP Status report 1/2005, Norwegian Meteorological Institute, Oslo, Norway. Retrieved from http:// www.emep.int.

UBA (2004). Manual on methodologies and criteria for modelling and mapping of critical loads and levels and air pollution effects, risks and trends. Umweltbundesamt, Dessau, Germany. Retrieved from http://www. icpmapping.org. 